\title{
Morphological and Chemical Analysis of Fe-doped Nanotitanates
}

\author{
A. M. L. M. $\operatorname{Costa}^{1}$, B. A. Marinkovic ${ }^{1}$, D. J. Smith $^{2}$, S. Paciornik ${ }^{1}$ \\ ${ }^{1}$ Departament of Materials Engineering/Pontifícia Universidade Católica do Rio de Janeiro, RJ - Brazil \\ ${ }^{2}$. Department of Physics/Arizona State University, Tempe - United States of America.
}

Nanostructured titanate is a promising semiconductor for applications in photoinduced processes such as energy conversion and degradation of pollutants [1]. The process is based on the generation of electronhole pairs due to absorption of energy identical to the semiconductor band gap. The created pair can be used to generate energy or to allow catalyst reactions. However, the titanate has a large band gap energy limiting the absorption energy to the ultraviolet range. Current studies have been carried out to increase this absorption range introducing Fe to decrease its band gap [2,3].

Generally, the doping of titanate occurs after its production, where $\mathrm{TiO}_{2}$ polymorphs (anatase, rutile or brookite) are used as precursor and the synthesis product is mixed with a Fe solution. To achieve synthesis and doping in a single step, an alternative way was proposed, starting from a Fe-Ti based sand via alkaline hydrothermal synthesis route.

To produce the Fe-doped titanates the Fe-Ti sand was mixed with $10 \mathrm{~mol}$. $\mathrm{L}^{-1} \mathrm{NaOH}$ aqueous solution in a Teflon vessel in a high pressurized stainless reactor (Bergoff). To obtain different morphologies the synthesis was carried out at $150^{\circ} \mathrm{C}$ and $190^{\circ} \mathrm{C}$. Transmission electron microscopy (TEM/STEM) was used to characterize the structure and morphology, and analytical electron microscopy (AEM) was used to evaluate the Fe presence in the products. The instruments used were a JEOL JEM 2100F and a JEOL JEM 4000EX, operating at 200 and $400 \mathrm{kV}$, respectively. Optical properties were measured using UVVIS absorption using diffuse reflectance spectroscopy (DRS) in a Cary 500 Scan UV-VIS-NIR (Varian) spectrophotometer.

Fig. 1a shows a BF/STEM image of agglomerated nanosheets formed at $150^{\circ} \mathrm{C}$. According to the elemental mapping of these nanosheets (Fig. 1b and 1c) $\mathrm{Fe}$ and $\mathrm{Ti}$ ions are present throughout the structure (Ti green and Fe yellow). Comparing with conventional Fe doped titanates, and based on the similarity of ionic radii of $\mathrm{Fe}^{+3}(0.785 \AA)$ and $\mathrm{Ti}^{+4}(0.745)$, the $\mathrm{Fe}$ atoms probably occupy the $\mathrm{Ti}^{+4}$ sites forming a similar structure. Fig. 2 a presents the nanoribbons formed at $190^{\circ} \mathrm{C}$. The mapping in Fig. $2 \mathrm{~b}$ shows that this morphology is formed only of $\mathrm{Ti}$ ions. This can be confirmed by a line scan across the same nanoribbon (Fig. 2c). Fig. 3a shows agglomerated nanoribbons with hematite nanocrystals attached (Fig. 3b). Fig 4 presents the DRS spectra showing the high absorption of these titanates in the visible range, where the sheets present higher absorption rates than the ribbons.

[1] O. Carp, C.L.Huisman, A. Reller, Progress in Solid State Chemistry 32 (2004) 33.

[2] E. Morgado Jr. et al, Mat. Chem. Phys. 126 (2011) 118.

[3] X. Ding, X.G. Xu, Q. Chen, L-M Peng, Nanotecnology 17 (2006), 5423.

[4] This research was supported by Brazilian government through CAPES, CNPq and FAPERJ agencies. 

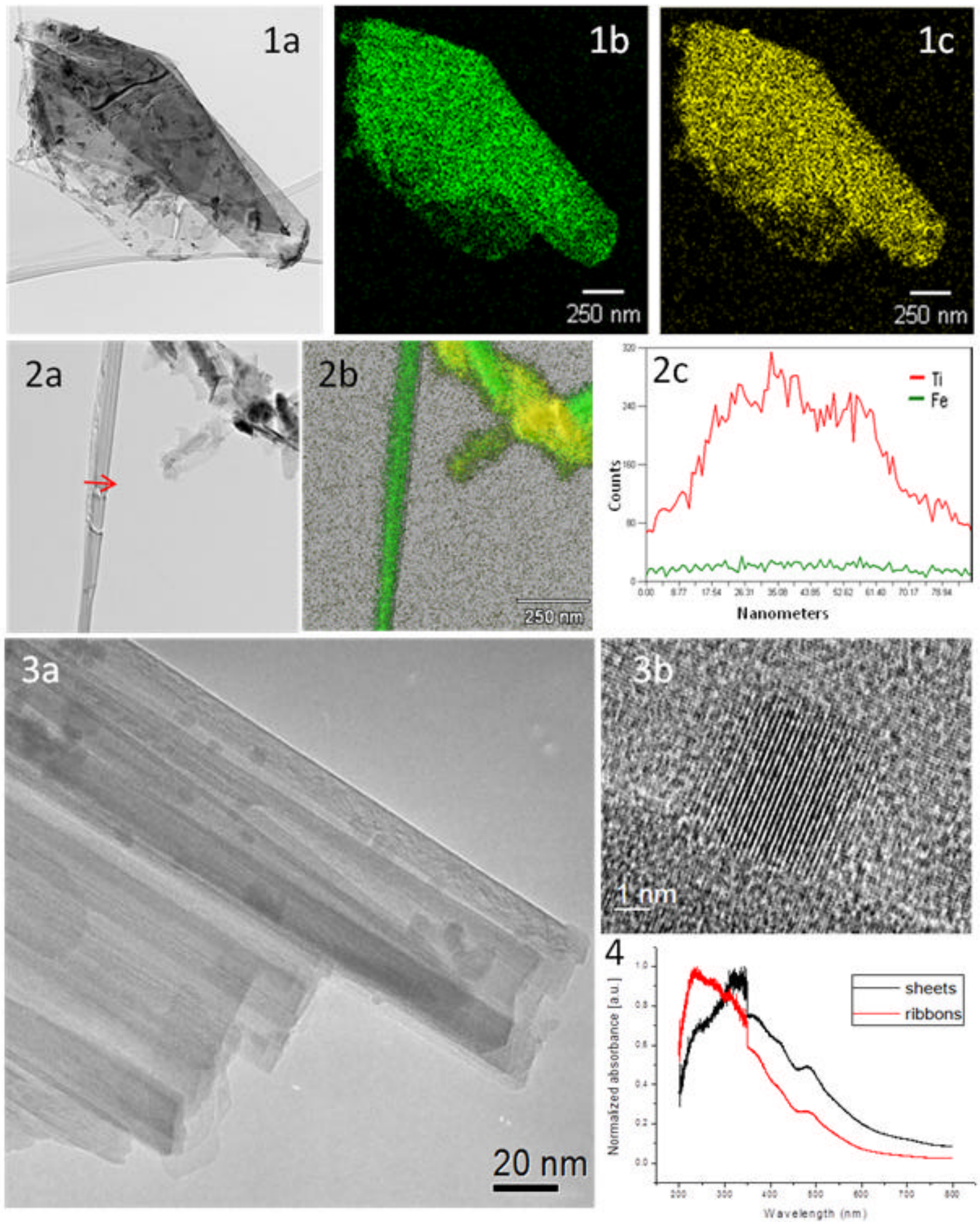

Figure 1 (a) STEM image of agglomerated nanosheets formed at $150^{\circ} \mathrm{C}$; Elemental mapping of (b) $\mathrm{Ti}$ and (c) Fe. Figure 2 (a) Nanoribbons and nanosheets synthesized at $190^{\circ} \mathrm{C}$; (b) Overlapped $\mathrm{Ti}$ and $\mathrm{Fe}$ mapping presents a mix of $\mathrm{Fe}$ and $\mathrm{Ti}$ in the sheet while the ribbon is formed only by Ti. (c) Line scan of the arrow in 2a. Figure 3 (a) shows agglomerated nanoribbons with nanoparticles of hematite attached formed at $190^{\circ} \mathrm{C}$; (b) HREM image of a hematite nanoparticle. Figure 4 is a DRS spectrum indicating high absorption in the UV-VIS range. 